\title{
Warum trägt Cundrî ein brûtlachen von Gent?
}

\author{
Höfische Mode zwischen den Kulturen
}

ANNA KARIN

\begin{abstract}
Fashion is an important part of medieval culture and courtly ceremonial. In a society that is organized to a significant degree by visibly displaying one's social status, fashion is one of the most relevant indicators of social distinction, of in-and exclusion. Therefore, the description of characters and their clothes is one of the typical devices in courtly literature to provide an impression of the status and virtues of these characters, their development and the current condition of the court and its members. The quality of courtly fashion depends on its design, the materials used and their origin, and the combination offoreign, exotic influences and familiar, regional elements. This article deals with the description of intercultural aspects of courtly fashion and its influence on the perception of characters, their development and the progression of the narration itself.
\end{abstract}

Title: Why Does Cundrî Wear ein brûtlachen von Gent? Courtly Fashion Between Cultures

Keywords: medieval court; courtly fashion; social distinction; characterization; notion of the Orient

\section{Höfische Mode und Kultur - eine Einführung}

Höfische Mode ist ein wesentlicher Bestandteil der höfischen Sach-, Zeremonial- und Kommunikationskultur und wird in entsprechend vielfältigen Kontexten in der höfischen Dichtung thematisiert und funktionalisiert. Am augenfälligsten und in der Regel auch ausführlichsten begegnet uns Mode seit der Schilderung von Didos Jagdgewand in Veldekes Eneit (vgl. 59,19-60,25) als Teil der Figurenzeichnung im Kontext von Gewand-, Körper- und insgesamt Figurendeskriptionen (vgl. Raudszus 1985: 63; Brüggen 1989: 39). Hierbei wird besonderer Wert auf das Prinzip der adaequatio gelegt, das basierend auf dem antiken Kalokagathia-Konzept das Äußere mit den inneren Werten in Relation setzt und sich in einer vollkommenen Übereinstimmung dieser Werte äußert. Die Bewertung dieser Relation ist im christlichen Denken erweitert worden, so dass man für das christlich-höfische Kalokagathia-Verständnis mit Kellermann (vgl. 2003: 110) festhalten kann: »Es gibt keine einfache Parallelität von schön und gut, sondern es gibt eine gute Seele, die sich im schönen Körper zeigt, und eine böse Seele, die sich im häßlichen Körper zur Anschauung bringt.« Die Kleidung ist ein wichtiger Teil dieses Prinzips, sie gehört zur Erscheinung einer Figur und entspricht 
im Idealfall ihrem Träger bzw. ihrer Trägerin ${ }^{1}$. Auf diese Weise dient sie der Lesbarkeit der höfischen Welt, indem sie beispielsweise soziale Zugehörigkeiten, die Wahrung der höfischen Ordnung, aber auch Ordnungsstörungen sichtbar macht, wenn die adaequatio in eine inadaequatio verkehrt wird. Kleidung wird somit zur Ausdrucksform einer Kultur als »symbolisch[e] Sinnordnung (Przybilski 2010: 7) und ist in diesem Kontext Teil der »soziale[n] Praxis« (Lüddemann 2010: 11). Aus einer Perspektive auf die vormoderne mittelalterliche Gesellschaft lässt sich Kultur mit Eagleton (2009: 51) »ungefähr als jenen Komplex von Werten, Sitten und Gebräuchen, Überzeugungen und Praktiken [zusammenfassen], die die Lebensweise einer bestimmten Gruppe ausmachen.« Für die »ritterlichhöfische Idealkultur« (Paravicini 2011: 5) äußert sich dies in einem hohen Grad an Konventionalisierung, so dass sich für die höfische Kultur durchaus von einer Leitkultur mit »klare[n] sozio-moralischen Leitlinien « (Leggewie/Zifonun 2010: 15) sprechen lässt. Gerade anhand der Kleidung als Materialisierung kultureller Vorstellungen und Werte zeigt sich der hohe ordnungsregulierende Symbolcharakter in einem dynamischen Spannungsfeld aus Disziplinierung und Alterität, Exklusion und Inklusion deutlich.

In der Literatur ist die Darstellung höfischer Mode neben figurenbezogenen Gewanddeskriptionen auch besonders für den Bereich des höfischen Zeremonialhandelns von Relevanz; hier wird »die gesellschaftliche Bedeutung der Mode« (Brüggen 1989: 124) in der Interaktion besonders augenfällig. Dies betrifft etwa die Ausstattung und teilweise eigenhändige Neueinkleidung der Gäste sowie das Herrichten der Hofgesellschaft für den Empfang der Gäste im Rahmen des Begrüßungszeremoniells. Auch das Verschenken von Kleidung, etwa im Kontext der Schwertleite, und ihre Vergabe als Botenlohn und Minnepfand sind Bestandteil des höfischen Zeremoniells (vgl. ebd.: 124-140).

Eine der wesentlichen Aufgaben höfischer Mode ist die soziale Distinktion ihrer Träger (vgl. Dinges 1993: 90-92; Kraß 2006: 158). Die Abgrenzung zu den anderen Ständen erfolgt gerade durch den Umstand, dass die höfische Kleidung nicht rein pragmatisch und alltagstauglich gestaltet ist, sondern der Repräsentation und Prachtentfaltung dient. Dies wird einerseits erreicht durch wertvolle Materialien und Herstellungsmerkmale wie »erlesene Stoffe und Pelze, kostbare und aufwendige Verzierungen und eine auffällige Farbigkeit « (Brüggen 1989: 47), andererseits durch die Fertigungsweise, die sich in einem hohen Materialverbrauch manifestiert, der den Überfluss der Gesellschaft versinnbildlicht und sich etwa in »langen Schleppen, weiten Ärmeln und gebogenen Schnabelschuhen « (Kraß 2006: 158) offenbart. Die Bedeutung und Wirkung der Mode ist immer wieder eine Frage von Prestige und Darstellung, aber auch von Zuordnung: Denn sie dient ihren Trägern dazu, sich gerade auch durch das Abgrenzen von anderen Schichten ihrem eigenen Stand zuzuordnen, dies wiederum wird durch ein Spiel aus höfischen und originär gerade nicht höfischen Elementen ermöglicht, durch ein Wechselspiel der Orientierung an höfischen Maßstäben und exo-

1 Im Folgenden werde ich aus Gründen der Einfachheit stellvertretend für beide Geschlechter von iTräger، sprechen. 
tischen Materialien. Simmel (1995: 14) beschreibt die Vorliebe für importierte Waren in seiner Philosophie der Mode als gemeinschaftsstiftend:

Tatsächlich scheint der exotische Ursprung der Mode den Zusammenschluß der Kreise, auf den sie angelegt ist, mit besonderer Stärke zu begünstigen; grade dadurch, daß sie von außen kommt, schafft sie jene besondere und bedeutsame Form der Sozialisierung, die durch die gemeinsame Beziehung zu einem außerhalb gelegenen Punkte eintritt.

In der höfischen Darstellung liegt dieser sozialisierende Faktor gerade in der Verbindung von Fremdem mit Eigenem, denn das Exotische wird durch eigene kulturelle Vorstellungen und Vorlieben überformt, angepasst und ihnen gemäß verarbeitet. Interkulturalität in der Mode äußert sich auf diese Weise in einem besonderen Spannungsverhältnis, in dem das Fremde zum Eigenen wird, das aber weiterhin fremd bleiben muss, um die Anforderungen des Exotischen erfüllen zu können und somit den Status des Höfischen zu betonen. Sieburg (2011: 13) konstatiert für die mittelalterliche deutsche Literatur, dass diese sich »unter maßgeblicher fremdkultureller Einflussnahme bzw. Orientierung « entwickelt dieses Bild spiegelt sich auch in den Modebeschreibungen wider, die davon leben, dass sie »Relationen des Kontrastes [...] oder auch der Komplementarität« (ebd.) in ihrer Darstellung miteinander verbinden.

Im Folgenden sollen die in der höfischen Mode miteinander verknüpften Vorstellungen verschiedener Kulturräume dargestellt und an ausgewählten Beispielen illustriert werden, wie sich die interkulturelle Darstellung der Mode auf die Figurenzeichnung, aber auch auf die Entwicklung der Narration auswirken kann.

\section{Mode als Vermittler kUltureller Vorstellungen}

Mit der literarischen Illustration höfischer Mode korreliert die Darstellung von kulturellem Prestige, Vorbild, aber auch Vorurteil. Grundsätzlich lassen sich zwei große Einflussbereiche ausmachen, die für die Qualität und Exklusivität der geschilderten Gewänder stehen: auf der einen Seite die an realhistorischen Gegebenheiten orientierte christlich-höfische Welt, auf der anderen Seite eine Welt des Fremden, Exotischen, Heidnischen, insbesondere der orientalische und asiatische Raum und in Verknüpfung mit diesem häufig phantastische Vorstellungen.

Eine Sonderstellung der Vorbildwirkung und des Einflusses auf die mittelhochdeutsche Literatur nicht nur im Bereich der Mode lässt sich für die französische Adelskultur nachweisen (vgl. Brüggen 1989: 194; vgl. insgesamt Sieburg 2011). So fallen zahlreiche Textstellen ins Auge, die den Zuschnitt der Kleider nach französischem Muster, also »die Körperformen nachzeichnend « (Brüggen 1989: 194; vgl. für eine Übersicht ebd.: 194f.), betonen. Dass auf diese Weise nicht nur die höfischen Qualitäten der Kleidung, sondern auch die ihrer Träger 
betont werden, wird in zwei sehr ausführlichen Gewanddeskriptionen deutlich, die als überbordende und zum Teil durchaus auch verstörende Schönheitspreise die Wirkung ihrer Trägerinnen auf die Hofgesellschaft in Szene setzen. So wird in Hartmanns von Aue (2007) Erec der genau angemessene Zuschnitt von Enites Obergewand nach französischem Vorbild lobend hervorgehoben, als Enite von Königin Ginover vor ihrer Einführung an den Artushof neu eingekleidet und für die Hofgesellschaft vorbereitet wird:

daz hemde si bedahte,

daz man'z loben mahte,

mit einem rocke wol gesniten

nâch kerlingischen siten,

weder z'enge noch ze wît [.....$^{2}$ (Er. 1544-1548)

Als eine der eindrucksvollsten Gewanddeskriptionen kann sicher Isoldes Auftritt am Hoftag von Weisefort in Gottfrieds von Straßburg Tristan gelten. Die descriptio veranschaulicht das Ideal von Kleidung, Figur und Präsentation und liest sich als eine Evokation von Höfischkeit in einer »perfekte[n] Synthese aus angeborenen Qualitäten und höfischer Kleidung « (Kellermann 1999: 43). Auch hier darf der Verweis nicht fehlen, dass Isoldes roc unde mantel [...] / in dem snite von Franze $^{3}$ (Tr. 10905f.) gestaltet sind (vgl. auch Kraß 2006: 159). Beide Deskriptionen sind an Textstellen zu finden, die die öffentliche Restitution der höfischen Ordnung behandeln.

Neben solchen expliziten Hinweisen auf das französische Vorbild belegen vor allem auch zahlreiche Entlehnungen den »deutsch-französische[n] Kulturtransfer « (Sieburg 2011: 24) im Kontext der höfischen Mode. Brüggen (vgl. 1989: 192f., dort auch das Glossar 202-293) ermittelt diverse entlehnte Termini aus dem Fachwortschatz der Textilfertigung, wie z.B. furrieren, »unterfüttern «, oder auch solche der Kleiderbezeichnung, wie blîalt, »Obergewand «, oder die als tassel bezeichnete Mantelschließe.

Auch die verwendeten Materialien geben Auskunft über kulturelle Vorstellungen, denn oft wird die Herkunft der Materialien angegeben, um deren Qualität, Exklusivität und Kostbarkeit herauszustellen. Gerade für die Schilderung der Stoffe gilt, dass diese häufig mit realhistorischen Orten verbunden werden, die bekannt für ausgezeichnete Gewebe sind. Für Wollstoffe ist das der europäische Raum. An erster Stelle steht Flandern als Produktionsort, wobei insbesondere die Städte Gent und seit der zweiten Hälfte des 13. Jahrhunderts auch Ypern

2 | "Über das Hemd zog sie / so, daß man es loben kann, / ein schön geschnittenes Kleid / nach französischer Mode, / es war nicht zu eng und nicht zu weit [...]“; Übersetzung von Held in Hartmann von Aue (2007). Den Terminus hemde sollte man passender mit "Unterkleid " übersetzen.

$3 \mid$ "[E]in Kleid und einen Mantel [...]/ nach französischer Mode“; hier und im Folgenden stammen alle Übersetzungen aus dem Tristan von Haug in der Ausgabe Gottfried von Straßburg (2012). 
als Ausweis für die besondere Qualität der Stoffe stehen (vgl. ebd.: 49f.; Raudszus 1985: 174). So trägt etwa die Gralsbotin Cundrî ein brûtlachen von Gent ${ }^{4}$ (Parz. 313,4). Neben Flandern spielt auch England eine wichtige Rolle: Für die Herkunft des besonders wertvollen Scharlachs aus England ermittelt Brüggen (vgl. 1989: 5of.) acht Belege 5 und folgert, dass diese Angaben »mit dem wirtschaftsgeschichtlichen Faktum in Verbindung stehen, daß für die Scharlachproduktion im Mittelalter nur erstklassige, und das bedeutete damals englische, Wolle verwendet wurde.« London ist schon seit dem 9. Jahrhundert als Fertigungsort für Scharlach und - dies mag eine kulturelle Besonderheit sein - Regenmäntel belegt, beide Handelsgüter waren »wichtige [...] Exportartikel des Landes« (Raudszus 1985: 174).

Ungemein wertvoller und exotischer als die Wollstoffe sind die feinen Seiden, und dies spiegelt sich auch in den Geschichten, die über ihre Herkunft erzählt werden. Die Exotik, insbesondere die Erwähnung der orientalischen Stoffherkunft, wird immer dann »in fast topischer Frequenz« (Kraß 2006: 159) ins Spiel gebracht, wenn der Akzent auf der Kostbarkeit der Kleidung liegt. Neben dem Orient wird auch Asien in den Epen als Herstellungsort für Seiden erwähnt (vgl. Brüggen 1989: 54), dies entspricht den historischen Exportbedingungen, denn Seide wurde zunächst in China hergestellt und "gelangte auf der sogenannten >Seidenstraße < an die Reichsgrenzen Chinas, wo sie nur von einzelnen arabischen Karawanen in Empfang genommen werden durften.« (Raudszus 1985: 176) Insbesondere Syrien kam eine zentrale Rolle für die Verarbeitung und den Export der Seide »nach Byzanz und in die Mittelmeerländer« (Kania 2010: 44) zu. Nach und nach fasste die Seidenproduktion auch in Norditalien Fuß und erreichte dort »ihre größte Blütezeit im 13. und 14. Jahrhundert« (ebd.). Obwohl zur Entstehungszeit der hochhöfischen Epik die Seidenherstellung also schon im europäischen Raum vertreten war, ${ }^{6}$ wird in der höfischen Literatur vor allem auf die orientalische Herkunft der Seiden eingegangen. ${ }^{7}$ Dies hängt mit der Assoziation des sagenhaften Reichtums des Orients zusammen. So hält Müller (o. $J .:$ 2) für das literarische Orientbild fest, »daß nach damaliger Meinung fast alles, was gut, teuer, raffiniert und luxuriös war, aus dem Orient stammte, nämlich aus den arabischen Ländern, aus Persien, der indischen Halbinsel oder gar dem fernen Osten.« Begehrte Luxusgüter waren etwa »Stoffe [...], Edelsteine, Gewürze, Parfums [...] [und] Waffen« (ebd.).

4 | "Genter Brauttuch«; hier und im Folgenden stammen alle Übersetzungen zum Parzival von Knecht in der Ausgabe Wolfram von Eschenbach (2003).

5 | Fünf dieser acht Belege stammen aus dem Demantin Bertholds von Holle, was den Wirkungsgrad natürlich einschränkt.

6 | Insbesondere die chinesische Seide galt aber lange als höherwertig (vgl. Raudszus 1985: 176f.).

7 | Namentlich werden etwa allgemein Arabien und konkret Persien, die Türkei, Lybien, Alexandrien und Syrien genannt (vgl. hierzu mit ausführlichen Belegstellen Brüggen 1989: 55). 
Neben dem in den Kleiderbeschreibungen vermittelten Bild des reichen Orients ist es aber vor allem das Faszinosum des Fremden, des Skurril-Phantastischen, das gleichermaßen die Imagination von ungemein ausgefallenen Stoffen wie das einer dem »vertrauten Eigenen extrem entgegengesetzte[n] Welt« (Mitsch 1992: 73) transportiert, wenn Besonderheiten der Stoffe mit wundersamen Herkunftsgeschichten versehen werden. Der Stoff von Gahmurets Waffenrock ist z.B. »aus Gold gewirkt, welches die Araber den Greifen entwenden, die es mit ihren Klauen aus einem Fels des Kaukasus reißen « (Brüggen 1989: 53). Daneben sind es oft Figuren aus orientalischen Ländern, die Stoffe tragen, die von Salamandern im Feuer gewebt werden (vgl. ebd.: 53f.). So trägt etwa Feirefiz einen geradezu unbezahlbaren Waffenrock, der sich neben dem Besatz mit kostbaren Edelsteinen durch dieses außergewöhnliche Gewebe auszeichnet (Parz. 735,24-27):

ime berge ze Agremuntîn

die würme salamander

in worhten ze ein ander

in dem heizen viure. ${ }^{8}$

Die Vorstellung dieser Stoffproduktion geht auf den auf etwa $116_{5}$ datierten Brief des Priesterkönigs Johannes zurück; es könnte sich bei dem Gewebe um seit der Antike bekannte feuerfeste Fasern wie Asbest handeln (vgl. Schmitz 2012: 53f.). Auch Feirefiz selbst betont die Feuerfestigkeit des Gewebes (Parz. 812,21-23):

wan mîn cursît salamander,

aspindê mîn schilt der ander,

ich waere verbrunnen von der tjost. ${ }^{9}$

Bei dem Berg, in dem der Stoff gewebt werden soll, handelt es sich wahrscheinlich um den Vulkan Ätna (vgl. Schmitz 2012: 54; Brüggen 1989: 54). In Wirnts von Grafenberg Wigalois hingegen wird die Wirkstätte der Salamander in Asien vermutet (vgl. ebd.).

Das interkulturell Reizvolle an der höfischen Mode ist, dass oft gerade die Elemente unterschiedlicher Provenienz miteinander verbunden werden, indem etwa ein Angehöriger des Hofes ein Kleid aus orientalischer Seide in französischem Zuschnitt trägt oder ein exotischer Träger in europäische Stoffe gekleidet ist. Wie sich dies auf die Figurenzeichnung auswirken kann, soll nun an Tristan, Gahmuret und Cundrî nachgezeichnet werden.

8 | "In einem Berg in Agremuntîn leben Salamander - die gehören zu den Schlangen -, und die webten in der Hitze des Feuers diesen Stoff."

9| "Wäre nicht mein Kursît aus Salamander gewesen und dazu mein Schild aus Aspindê, so hätte ich verbrennen müssen von der Tjost." 


\section{Fremde Träger und Träger des Fremden}

Die distinktive Funktion von Kleidung soll eine klare Zuordenbarkeit ihrer Träger gewährleisten. Während in vielen Schilderungen der Status, die (höfische/ nichthöfische) Sphäre, die descriptio der Träger selbst und die Beschreibung des Gewandes übereinstimmen, kommt es immer wieder auch zu Darstellungen, in denen die Figuren oder die Situation, in der sie sich befinden, durch die Kleidung kontrastiert werden. Während in vielen Deskriptionen die von Simmel (vgl. 1995: 14) bemerkte Eigenschaft des Fremden in der Mode zum Ausdruck kommt, eine gemeinsame (in unserem Fall höfische) Identität stiften zu können, kann die Erwähnung des Exotischen aber auch gerade dazu dienen, die Fremdheit ihres Trägers zu unterstreichen. Die Nennung einer orientalischen Provenienz eines Stoffes kann also gleichermaßen eine ex- wie inkludierende Funktion haben und ist zwar als grundsätzlich topischer Bestandteil der höfischen Kleiderkultur zu verstehen, der aber durch eine Übersteigerung zu einer Art Überfremdung führen kann.

Ein Beispiel für eine Gewanddeskription, in der der interkulturelle Aspekt der Kleidung sich als gerade nicht verbindend, sondern abgrenzend erweist, ist die Beschreibung des jungen Tristan, der nach seiner Entführung ausgesetzt wird und sich in der Wildnis zurechtfinden muss. Bedenkt man, dass Kleiderbeschreibungen, die die höfische Pracht inszenieren, in der Regel in repräsentativen Kontexten am Hof situiert sind, wird deutlich, wie ungewöhnlich es ist, die erste Gewanddeskription Tristans genau außerhalb der höfischen Welt an einem kulturfernen Ort zu platzieren. Beschrieben werden Tristans roc unde mantel $^{10}$ (Tr. 2534) aus Pheller, einem hochwertigen Seidenstoff, der sich einerseits durch seine Kostbarkeit auszeichnet, andererseits aber dadurch, dass er an gewürhte wunderlîch ${ }^{11}$ (ebd.: 2536) ist. Mit wunderlîch ist gleichermaßen die wunderbare wie die seltsam-außergewöhnliche Wirkung des Kleidungsstückes (auf den Betrachter oder in der Beschreibung auf den Rezipienten) angesprochen, deren Ursprung direkt im Anschluss auf den Exotismus des Gewandes zurückgeführt wird, wie der Text gleich in mehrfacher Weise betont. Nicht nur ist er von Sarazenen hergestellt worden, sondern auch ein typisches Element des Gewandschmucks, der Besatz mit Borten, erfolgt hier nach heidnischer Art und hebt sich so vom Bekannten ab (ebd.: 2537-2546):

er was von Sarrazînen

mit cleinen bortelînen

in vremdeclîchem prîse

nâch heidenischer wîse

wol underworht und underbriten

und was der alsô wol gesniten

nâch sînem schœnem lîbe,

10 | "Rock und einen Mantel«; der Terminus roc bezeichnet das Obergewand.

11 | "[W]underbar gewoben". 
daz von manne noch von wîbe

enwurden edeler cleider nie

baz gesniten danne die. ${ }^{12}$

Die orientalische Provenienz des Stoffes wird wörtlich mit seiner Fremdartigkeit in Relation gesetzt und diese Fremdartigkeit korreliert mit der ebenso hervorgehobenen prachtvollen Schönheit der Gewandung, die sich dadurch auszeichnet, dass sie Tristan im wahrsten Sinne auf den Leib geschneidert ist. Auch ihre grüne Farbe und ihre Unterfütterung mit Hermelin wird im Folgenden hervorgehoben, wodurch zwei topische Elemente der höfischen Gewandbeschreibung bedient werden (vgl. Brüggen 1989: 59-63). Die Kleidung erscheint hier als zugleich höfisch und fremd. Insgesamt bildet sie einen Kontrast zur Situation des Helden und erweist sich als der kulturfernen, wilden Umgebung nicht adäquat, da ihr jede Alltagstauglichkeit fehlt. Huber (2007: 135) hält zu Tristans Erscheinung fest: »Dieser Aufzug ist edel, wertvoll, kulturell raffiniert, aber auch befremdend.« Dieser Kommentar zu Tristans Kleidung könnte ebenso gut ein Kommentar über den Protagonisten selbst sein, der sich im weiteren Handlungsverlauf durch seine kulturelle Raffinesse, seine Höfischkeit, aber auch seine Befremdlichkeit und vor allem immer wieder Fremdheit auszeichnet, denn Tristan wird der besondere Fremde bleiben. Gleichermaßen offenbart Tristans Umgang mit seiner Kleidung bereits eine Eigenschaft, die ihn grundsätzlich auszeichnet (Tr. 2557-2562):

\author{
under sînen gürtel zôher \\ sînen roc ein lützel hôher; \\ den mantel want er in ein \\ und leite in ûf sîn ahselbein \\ und streich ûf gein der wilde \\ durch walt und durch gevilde. ${ }^{13}$
}

Tristan ist in der Lage, sich an Situationen anzupassen und dies auch jenseits der höfischen Welt sowie jenseits der höfischen Norm, von der er sich, wenn nötig, distanzieren kann.

Auch der zeremonielle Umgang mit der Kleidung beeinflusst deren Lesbarkeit, gerade dann, wenn das Zeremoniell mit der descriptio in einen engen Kontext gesetzt wird. Ein Blick auf Gahmurets Waffenrock kann veranschaulichen, in wie vielfältiger Weise ein Kleidungsstück Aussagen über den Status seines

12 | "Sarazenen hatten inn / mit zierlichen Bändern / fremdartig und bewunderungswürdig / in heidnischer Manier / durchflochten und durchwoben, / und er war so vortrefflich / auf seinen schönen Körper zugeschnitten, / daß kein Mensch / vornehme Kleider jemals besser zugeschnitten hat / als diese hier."

13 | "Er zog unter dem Gürtel / seinen Rock ein wenig hoch; / den Mantel rollte er zusammen / und legte inn sich über die Schulter / und stieg flink über Halden und durch Gehölz / in die wilde Höhe empor." Auch hier bezeichnet der roc das Obergewand. 
Trägers zulässt, aber auch über die Vorstellung einer interkulturellen Verbindlichkeit höfischen Zeremonialhandelns. Hier verbinden sich zwei große Komponenten, auf die die Beschreibung verweist - Gahmurets ganz im wörtlichen Sinn inter-kultureller Status (denn er verbleibt fortan immer zwischen den Kulturen) und seine Kampfeskraft: Ein Jahr, nachdem Gahmuret Belakane heimlich verlassen hat, begegnet er auf See den Boten des Schotten Friedebrant, der zuvor Zazamanc belagert hatte und nun als Wiedergutmachung für den kriegerischen Einfall Boten mit einer überaus kostbaren Rüstung zu Belakane schickt. Hier wird zunächst die Vorstellung vermittelt, dass die Entschädigung eines vormaligen Gegners über kostbare Gegenstände wie eine Rüstung, die im weiteren Sinne der Kleidung zugerechnet werden kann, ein sowohl an den europäischen wie orientalischen Höfen akzeptiertes Modell der Schadensregulierung ist und als Teil der Sachkultur einen in beiden Kulturen gleichermaßen festen Platz hat. Noltze (1995: 118) ist zuzustimmen, wenn er für die Gahmuret-Bücher festhält: »Abendund Morgenland erscheinen als verbundene Welten letztlich einer höfisch-ritterlichen Kultur, obwohl die Andersartigkeit mehrfach thematisiert wird «.

Gahmuret nimmt die Rüstung an sich und gibt den Boten das Versprechen, diese seiner Frau zu überbringen, swenne er koeme ze ir ${ }^{14}$ (Parz. 58,19; vgl. auch ebd.: 57,29-58,19). Bekanntlich wird er das nicht tun. Stattdessen trägt er eben diese Rüstung am Vorabend des Turniers von Kanvoleis, wo noch einmal betont wird, dass sie für Belakane bestimmt ist (vgl. ebd.: 70,13-18). Besonders hervorgehoben wird ein Diamanthelm, auf den Gahmurets Wappen, ein edelsteinverzierter Anker, befestigt wird. Der Schild, ebenfalls Teil der Rüstung, ist explizit aus golde von Arabî $\hat{\imath}^{15}$ (ebd.: 70,28) mit hohem Gewicht gefertigt und wird durch einen Anker aus Zobelpelz geziert. Das Prunkstück von Gahmurets kriegerischem Aufzug ist jedoch sein Waffenrock, der sich durch eine Mischung klassischer Elemente der höfischen Kleiderbeschreibungen und eine ausgefallene Exotik auszeichnet und auf den Status Gahmurets als Reisender zwischen den Kulturen hindeutet. Eingeleitet wird die descriptio des Waffenrocks durch einen Verweis auf die verschwenderische Materialfülle (ebd.: 71,7): Sîn wapenroc was harte wit. ${ }^{16}$ Dies manifestiert sich schon in einer Länge, die bis auf die Treppe hinunterreicht. Die größte Besonderheit des Waffenrocks ist allerdings seine ungeheure Leuchtkraft, mit der wiederum ein typisches Merkmal höfischer Gewandbeschreibungen zitiert (vgl. Brüggen 1989: 69f.) und in übersteigernder Intensität geschildert und erklärt wird. Der Glanz der Kleidung ist gemäß des adaequatio-Prinzips ein wichtiger Bestandteil der Deskriptionen: »[I]m glanzvollen Äußeren [sollen sich] die inneren Qualitäten des Trägers manifestieren« (Raudszus 1985: 106). Neben Vergleichen mit dem Feuer und dem Hinweis, dass kranke Augen ob des Glanzes schmerzen könnten, ist es nun gerade die Verknüpfung mit der fremdartigen Herkunft des Stoffes, die eine regelrech-

14 | "[S]obald er wieder zur Königin käme."

15 | "Gold aus Arabien[.]"

16 | "Sein Waffenrock war reich und weit[.]" 
te Übersteigerung von Wert, Strahlkraft, Pracht und eine exotisch-höfische Inszenierung bewirkt (Parz. 71,17-28):

mit golde er gebildet was, daz zer muntâne Kaukasas ab einem velse zarten grîfen clâ, die ez dâ bewarten und ez noch hiute bewarent. von Arâbî liute varent: die erwerbent ez mit listen dâ (sô tiurez ist ninder anderswâ) und bringentz wider ze Arâbî, dâ man diu grüenen achmardi wurket und die pfellel rîch. ander wât ist der vil ungelîch. ${ }^{17}$

Hierbei werden Kulturvorstellungen vermittelt, deren aktuelle Gültigkeit durch die zeitliche Einordnung, und ez noch hiute bewarent, sowie den Wechsel ins Präsens betont wird: Der Stoff ist mit Gold durchwirkt, welches Greifen im Kaukasus aus einem Berg geschlagen haben. Dieses wertvollste Gold entwenden Araber den Greifen mit listen und bringen es nach Arabien, das hier als Produktionsort zum einen für den besonderen, durch seine grüne Farbe gekennzeichneten Achmardi (vgl. Brüggen 1989: 268) ${ }^{18}$, zum anderen für Seiden allgemein genannt wird. Neben den Hinweisen auf den Stoff erfolgt quasi nebenbei eine Charakterisierung der Araber als listig, wobei das in diesem Kontext nicht negativ konnotiert sein muss, sehr wohl aber auf das möglicherweise gefährliche Geschick der Araber verweist, die offenbar die Greifen immer wieder überwinden können. Das Bild des Fremden setzt sich hier mit den Greifen aus Vorstellungen, die der altorientalischen Mythologie entspringen, sowie realhistorischen Gegebenheiten wie der Seidenherkunft zusammen und führt überdies die besondere Geschicklichkeit der Araber in der Überwindung gefährlicher Kreaturen vor Augen. An dieser Stelle kommt nun wieder die Korrelation von Kleidung und Figur zum Tragen, denn die Fähigkeit, über die Greifen zu siegen, ist gewissermaßen auch dem Träger des Waffenrocks eingeschrieben: Das Feldzeichen der ersten Gegner Gahmurets ist der Greif, aufgeteilt in den Greifenschweif, reprä-

17 | "Es waren Bilder auf dem Stoff aus Gold von einer Sorte, das im Gebirge Kaukasas Greifen mit ihren Krallen von einem Felsen brachen; die waren nämlich da die Hüter dieses Goldes, und sie sind es noch heute. In Arabien gibt es Leute, die fahren dorthin und holen es sich mit viel Schlauheit - nirgends auf der Welt gibt es so teures Gold. Dann bringen sie es heim nach Arabien, wo man die grünen Achmardîstoffe webt und den edlen Phellel; kein anderes Gewebe kann sich mit denen messen."

18 | Raudszus (1985: 106) stellt fest, dass "die Farbe Grün konsequent zur Kennzeichnung Gahmurets verwendet wird; sie wiederholt sich nicht nur an seiner Kleidung, sondern auch an seiner gesamten Ausrüstung." 
sentiert durch vier Banner, unter denen kühne Rotten reiten, und das Vorderteil des Greifen als Wappen des Königs von Gascogne (vgl. Parz. 72,17-26). Letzterer wird von Gahmuret, der hier in Betonung seines bereits im Orient erstrittenen Ruhms als werde künec von Zazamanc ${ }^{19}$ (ebd.: 73,3) bezeichnet wird (vgl. hierzu auch Schotte 2009: 68), gefangen genommen. Gahmuret, das wird hier offenkundig, bleibt zwischen den Welten, und seine Kampfeskraft, sein Waffenrock, aber auch sein Titel machen deutlich, dass der Orient Gahmuret inhärent ist und gerade auch dies zu seinem Erfolg beiträgt.

Später wird es dann auch sein Waffenrock sein, den seine neue Frau Herzeloyde ersetzt: Nach den Kämpfen wird ihr dieser Waffenrock durch einen Pagen übergeben; an ihm erkennt sie, dass Gahmuret der Gewinner der Kämpfe ist (vgl. Parz. 82,3f.), aber auch, dass er durch diesen Waffenrock einer anderen Frau zugeordnet wird, was deutlich wird, wenn sie zum Waffenrock sagt: dich hat ein werdez wîp gesant / bî disem ritter in diz lant ${ }^{20}$ (ebd.: 81,25f.). In seiner Ehe mit Herzeloyde wird Gahmuret bei seinen Kämpfen keinen Waffenrock mehr über der Rüstung tragen, sondern insgesamt 18 weiße hemden (vgl. zur Übersetzung Anm. 2) Herzeloydes, also auf der bloßen Haut getragene Unterkleider, die Herzeloyde nach seiner Rückkehr wieder auf der Haut tragen wird (vgl. ebd.: 101,10-19). Der orientalische Waffenrock wird hier also durch ein erotisches, aber dennoch mit höfischer Symbolik aufgeladenes Minnepfand ersetzt, und dieses dient durchaus auch der Abgrenzung zu Gahmurets voriger Ehefrau. Gahmuret jedoch bleibt dem Orient verhaftet und findet dort in ein solches hemde gekleidet seinen Tod. Herzeloyde bleibt nur dieses blutige, zerfetzte hemde, das sie wieder auf der Haut tragen möchte - stattdessen wird das bluot Gahmurets, ${ }^{21}$ das ja an dem hemde klebt, stellvertretend für den bereits im Orient beigesetzten Helden im Münster bestattet (vgl. ebd.: 111,14-112,2). Gahmuret verbleibt somit auch im Tod zwischen Orient und Okzident, und so, wie ihm beide Kulturräume inhärent sind, bleibt auch er beiden Kulturräumen eingeschrieben.

Während bei den zwei hier untersuchten genuin höfischen Figuren Tristan und Gahmuret besonderer Wert auf die orientalische Herkunft der Stoffe gelegt wird, wird bei der Gralsbotin Cundrî genau gegenteilig verfahren. Ihre mit Tiervergleichen exemplifizierte Hässlichkeit repräsentiert ihre heidnische Abkunft von den Adamstöchtern und steht nicht nur im Gegensatz zu ihrer triw $[e]^{22}$ (Parz. 312,3) und Mitleidsfähigkeit, sondern wird auch durch ihre modisch-exquisite Kleidung kontrastiert, die ihre Zugehörigkeit zur höfischen Welt symbolisiert (vgl. ebd.: 312,2-314,10). Dies wird durch die Wahl ihrer Kleidungsstücke, die Herkunft der Stoffe, den Zuschnitt sowie die Farbigkeit bewirkt (ebd.: 313,1-13):

19 | "[D]er edle König von Zazamanc".

20 | "Das war eine edle Dame, die dich mit diesem Ritter in dies Land geschickt hat."

21 | Ebenso verhält es sich mit der tödlichen Waffe, dem sper, "Speer" (Parz. 111,20; $112,1)$.

22 | Hier wird auf den höfischen Wert der verbindlichen Treue, Loyalität und Aufrichtigkeit abgehoben. 
Diu maget witze rîche

was gevar den unglîche

die man da heizent bêâ schent.

ein brûtlachen von Gent,

noch plâwer denne ein lâsûr,

het an geleit der freuden schûr:

daz was ein kappe wol gesniten

al nâch der Franzoyser siten:

drunde an ir lîb was pfelle guot.

von Lunders ein pfæwîn huot,

gefurriert mit einem blîalt

(der huot was niwe, diu snuor niht alt),

der hieng ir an dem rücke. ${ }^{23}$

Zwei Kleidungsstücke werden hier eingehender beschrieben, Cundrîs Reisekappe und ihr Pfauenhut. Auffällig ist, dass alle Herkunftsbezeichnungen den europäischen Raum hervorheben, und dies, obwohl Cundrî sehr wohl auch Seide unter ihrer Reisekappe trägt und ihr Hut ebenfalls mit Seide unterfüttert ist. Erwähnt werden konkret der ja eigentlich im Vergleich zur Seide minderwertigere Wollstoff der Reisekappe, ein brûtlachen von Gent, der französische Zuschnitt des Reisemantels sowie die Herkunft des Pfauenhuts aus London. Die Orte, die hier genannt werden, sind solche, die in der europäisch-höfischen Modekultur großes Prestige besitzen (vgl. Brüggen 1989: 49-51). So wie Wolfram bei Cundrî mit den gängigen Elementen einer Schönheitsbeschreibung spielt (vgl. z.B. Schausten 2008: 462), parodiert er hier den gängigen Exotismus der Kleiderbeschreibungen, indem er gerade die europäische Provenienz der Kleidung betont und die der Seiden auslässt. Hierbei wählt er zudem eine für Kleiderbeschreibungen grundsätzlich ungewöhnliche Farbigkeit, denn Cundrîs Kappe ist blauer als ein Lasurstein $^{24}$ - hierdurch wird auf ungewöhnliche Weise der Wert der Farbe hervorgehoben, denn: »Der echte Lasurstein und die aus ihm hergestellte blaue Farbe waren neben dem Gold das wertvollste Material der mittelalterlichen Kunst.« (Ploss 1962: 77) Vor allem aber hat das Blau in der christlichen Ikonographie (nicht nur) des Mittelalters einen festen Symbolwert, den auch Raudszus (1985: 128; vgl. auch Forstner 1977: 116f.) hervorhebt: »Blau symbolisiert in der mittelalterlichen Tradition ganz ungebrochen das Himmlische, ist Sinnbild für Dauer, Festigkeit und Treue und kennzeichnet als Farbattribut in der Ikonographie

23 | "Die junge Dame, die so gelehrt war, sah ganz anders aus als sonst die schönen Feinen. Genter Brauttuch, blauer als Lapislazuli, trug dieser Hagelschlag des Glücks: ein elegantes Cape nach französischem Schnitt; darunter trug sie feine Seide am Leib. Ein Pfauenhut aus Londres war mit golddurchwirkter Seide gefüttert, der Hut war neu, die Borte daran nicht von gestern, der hing ihr auf den Rücken."

24 | Schausten (2008: 480) hält fest, dass im Parzival "die Farben Schwarz, Weiß und Rot [dominieren]." 
häufig die Mutter Gottes.« Auf diese Weise wird Cundrî ebenso dem »sakralen Gralsbereich « (Raudszus 1985: 128) zugeordnet wie auch durch den Pfauenhut: Neben der Tatsache, dass Anfortas ebenfalls einen Pfauenhut trägt, ist der Pfau im Mittelalter wegen der jährlichen Erneuerung seines Gefieders ein »Symbol der Unsterblichkeit, der geistigen Erneuerung und der himmlischen Herrlichkeit« (ebd.; vgl. auch Forstner 1977: 116f., 230-232). Die descriptio Cundrîs, gerade durch die auffällige inadaequatio, verdeutlicht, dass Cundrî in jede der anzitierten Welten und Kulturen - den heidnischen Orient, die höfische Welt und die Gralsgemeinschaft - bis zu einem gewissen Punkt hineingehört und in diese integriert ist, gleichzeitig aber bleibt sie allen Welten fremd: Mit Gerok-Reiter (2006: 110) ist ihre Uneindeutigkeit das wesentliche Merkmal Cundrîs: Sie ist »zugleich hässlich und gut, unhöfisch und höfisch«. Dies ist kein auf Überwindung angelegter Zustand, wie man ihn etwa bei auf die inadaequatio hin zielenden Gewandschilderungen bei vorübergehend defizitären Adligen wie Enite, Jeschute (vgl. Parz. 256,11-257,25) oder Rual (vgl. Tr. 3994-4015) findet. Vielmehr ist es die Grundlage ihrer Mobilität und konstitutiv für ihre Funktion als Gralsbotin, die sich zwischen den verschiedenen Welten bewegen muss.

\section{FAZIT}

Mode ist, im Mittelalter wie heute, geprägt durch ganz unterschiedliche kulturelle Einflüsse, und so, wie die unterschiedlichen Kulturen die Mode beeinflussen, beeinflussen die Mode und ihre Darstellung wiederum das Bild, das von diesen Kulturen vermittelt und mit ihnen assoziiert wird. Wie gekonnt diese Interkulturalität von Mode von den höfischen Dichtern eingesetzt wird, um ganz unterschiedliche Effekte zu erreichen, konnte hier nur angedeutet werden: So können durchaus typische Elemente der Kleiderbeschreibungen in ihrer Lesbarkeit variieren und müssen kontextbezogen gedeutet werden; sie können eine interkulturelle Verbindung ebenso akzentuieren wie den Aspekt des Fremden, geradezu Gegenkulturellen. Dabei macht insbesondere das Spiel mit modischen Stereotypen einen Bruch mit denselben möglich: So wie Isoldes und Enites Gewandbeschreibungen sie als idealtypische, aber in ihrer Schönheit gefährliche Damen inszenieren, kennzeichnet Tristans Gewandung ihn als fremd, während Cundrî durch ihre Kleidung als nicht bloß fremd charakterisiert wird und Gahmuret als Grenzgänger zwischen dem Orient und dem Okzident erscheint. Die Lesbarkeit der Figuren und ihre Verortung in der höfischen Welt wird hierbei gerade nicht nur durch die topischen Elemente der Schönheitsbeschreibung, der Licht- und Farbmetaphorik und der Darstellung von Pracht und Reichtum erreicht, sondern besonders auch durch den oft eher als stereotypes Detail am Rande erscheinenden Hinweis auf die Provenienz von Schnitten, Stoffen und Gewandschmuck. 


\section{LITERATUR}

Brüggen, Elke (1989): Kleidung und Mode in der höfischen Epik des 12. und 13. Jahrhunderts. Heidelberg.

Dinges, Martin (1993): Von der "Lesbarkeit der Welt" zum universalisierten Wandel durch individuelle Strategien. Die soziale Funktion der Kleidung in der höfischen Gesellschaft. In: Saeculum 44, H. 1, S. 90-112.

Eagleton, Terry (2009): Was ist Kultur? Eine Einführung. Aus dem Engl. v. Holger Flissbach, München.

Forstner, Dorothea ( ${ }^{31977): ~ D i e ~ W e l t ~ d e r ~ c h r i s t l i c h e n ~ S y m b o l e . ~ M i t ~} 32$ Kunstdruckbildern. Innsbruck/München/Wien.

Gerok-Reiter, Annette (2006): Individualität. Studien zu einem umstrittenen Phänomen mittelhochdeutscher Epik. Tübingen/Basel.

Gottfried von Straßburg (2012): Tristan und Isold. Hg. v. Walter Haug u. Manfred Günter Scholz. Mit dem Text des Thomas, hg., übers. u. komm. v. Walter Haug. Bd. I. Berlin.

Hartmann von Aue (2007): Erec. Hg. v. Manfred Günter Scholz. Übers. v. Susanne Held. Frankfurt a.M.

Heinrich von Veldeke (1992): Eneasroman. Die Berliner Bilderhandschrift mit Übersetzung und Kommentar. Hg. v. Hans Fromm. Mit den Miniaturen der Handschrift und einem Aufsatz von Dorothea und Peter Diemer. Frankfurt a.M.

Huber, Christoph (2007): Merkmale des Schönen und volkssprachliche Literarästhetik. Zu Hartmann von Aue und Gottfried von Straßburg. In: Manuel Braun/Christopher Young (Hg.): Das fremde Schöne. Dimensionen des Ästhetischen in der Literatur des Mittelalters. Berlin/ New York, S. 111-141.

Kania, Katrin (2010): Kleidung im Mittelalter. Materialien - Konstruktion - Nähtechnik. Ein Handbuch. Köln/Weimar/Wien.

Kellermann, Karina (1999): Entstellt, verstümmelt, gezeichnet - Wenn höfische Körper aus der Form geraten. In: Iris Denneler (Hg.): Die Formel und das Unverwechselbare. Interdisziplinäre Beiträge zu Topik, Rhetorik und Individualität. Frankfurt a.M. u.a., S. 39-58.

Dies. (2003): Entblößungen. Die poetologische Funktion des Körpers in Tugendproben der Artusepik. In: Das Mittelalter 8, H. 1, S. 102-117.

Kraß, Andreas (2006): Geschriebene Kleider. Höfische Identität als literarisches Spiel. Tübingen/Basel.

Leggewie, Claus/Zifonun, Dariuš (2010): Was heißt Interkulturalität? In: Zeitschrift für interkulturelle Germanistik 1, H. 1, S. 11-31.

Lüddemann, Stefan (2010): Kultur. Eine Einführung. Wiesbaden.

Mitsch, Ralf (1992): Körper als Zeichenträger kultureller Alterität. Zur Wahrnehmung und Darstellung fremder Kulturen in mittelalterlichen Quellen. In: Burkhardt Krause (Hg.): Fremdkörper - Fremde Körper - Körperfremde. Kultur- und literaturgeschichtliche Studien zum Körperthema. Stuttgart, S. 73-109.

Müller, Ulrich (o. J.): Orient und Okzident in der europäischen Literatur des Mittelalters - Eine Einführung; online unter: https://www.uni-salzburg.at/fileadmin/ oracle_file_imports/544377.PDF [Stand: 1.9.2018]. 
Noltze, Holger (1995): bî den dûht in diu wîle lanc - Warum langweilt sich Gahmuret bei den Môren? (Zu Pz. 17,26). In: Dorothee Lindemann/Berndt Volkmann/KlausPeter Wegera (Hg.): bickelwort und wildiu mære. Festschrift für Eberhard Nellmann zum 65. Geburtstag. Göppingen, S. 109-119.

Paravicini, Werner (2011): Die ritterlich-höfische Kultur des Mittelalters. München.

Ploss, Emil Ernst (1962): Ein Buch von alten Farben: Technologie der Textilfarben im Mittelalter mit einem Ausblick auf die festen Farben. Heidelberg/Berlin.

Przybilski, Martin (2010): Kulturtransfer zwischen Juden und Christen in der deutschen Literatur des Mittelalters. Berlin/ NewYork.

Raudszus, Gabriele (1985): Die Zeichensprache der Kleidung. Untersuchungen zur Symbolik des Gewandes in der deutschen Epik des Mittelalters. Hildesheim / Zürich/New York.

Schausten, Monika (2008): Vom Fall in die Farbe. Chromophilie in Wolframs von Eschenbach ,Parzivalı. In: Beiträge zur Geschichte der deutschen Sprache und Literatur 130, H. 3, S. 459-482.

Schmitz, Michaela (2012): Der Schluss des Parzival Wolframs von Eschenbach. Kommentar zum 16. Buch. Berlin.

Schotte, Manuela (2009): Christen, Heiden und der Gral. Die Heidendarstellung als Instrument der Rezeptionslenkung in den mittelhochdeutschen Gralsromanen des 13. Jahrhunderts. Frankfurt a.M.

Sieburg, Heinz (2011): Plädoyer für eine interkulturelle Mediävistik. In: Zeitschrift für interkulturelle Germanistik 2, H. 1, S. 11-26.

Simmel, Georg (1995): Philosophie der Mode [1905]. In: Ders.: Gesamtausgabe. Hg. v. Otthein Rammstedt. Bd. 10. Frankfurt a.M., S. 7-37.

Wolfram von Eschenbach (2003): Parzival. Mittelhochdeutscher Text nach der sechsten Ausg. v. Karl Lachmann. Übers. v. Peter Knecht. Mit Einführungen zum Text der Lachmannschen Ausgabe und in Probleme der Parzivalı-Interpretation v. Bernd Schirok. Berlin/ New York. 
